\title{
Automated BRAin MASKing of Fetal Functional MRI
}

A PREPRINT

\author{
Saige Rutherford* \\ Department of Psychiatry \\ University of Michigan \\ Ann Arbor, MI \\ saruther@med.umich.edu
}

\author{
Pascal Sturmfels \\ Department of Electrical Engineering \\ and Computer Science \\ University of Michigan \\ Ann Arbor, MI
}

\author{
Mike Angstadt \\ Department of Psychiatry \\ University of Michigan \\ Ann Arbor, MI
}

\author{
Jasmine Hect \\ Merrill Palmer Skillman Institute \\ Wayne State University \\ Detroit, MI \\ Marion van den Heuval \\ Department of Cognitive Neuropsychology \\ University of Tilburg \\ Tilburg, The Netherlands

\section{Moriah Thomason} \\ Department of Child and Adolescent Psychiatry \\ New York University Langone \\ New York, NY
}

\author{
Jenna Wiens \\ Department of Electrical Engineering \\ and Computer Science \\ University of Michigan \\ Ann Arbor, MI
}

\author{
Dustin Scheinost \\ Department of Radiology \\ and Biomedical Imaging \\ Yale University School of Medicine \\ New Haven, CT \\ Chandra Sripada \\ Department of Psychiatry \\ University of Michigan \\ Ann Arbor, MI
}

July 12, 2019

\begin{abstract}
Fetal resting-state functional magnetic resonance imaging (rs-fMRI) has emerged as a critical new approach for characterizing brain development before birth. Despite rapid and widespread growth of this approach, at present we lack neuroimaging processing pipelines suited to address the unique challenges inherent in this data type. Here, we solve the most challenging processing step, rapid and accurate isolation of the fetal brain from surrounding tissue across thousands of non-stationary 3D brain volumes. Leveraging our library of 1,241 manually traced fetal fMRI images from 207 fetuses (gestational age 24-39 weeks, $\mathrm{M}=30.9, \mathrm{SD}=4.2$ ), we trained a Convolutional Neural Network (CNN) that achieved excellent performance across two held-out test sets from separate scanners and populations. Furthermore, we unite the auto-masking model with additional fMRI preprocessing steps from existing software and provide insight into our adaptation of each step. This work represents an initial advancement towards a fully comprehensive, open source workflow for fetal functional MRI data preprocessing.
\end{abstract}

Keywords fetal · fMRI · functional imaging · brain segmentation · deep learning · convolutional neural network · automated processing $\cdot$ open-source software

\footnotetext{
* corresponding author
} 


\section{Introduction}

Resting-state functional magnetic resonance imaging (rs-fMRI) has emerged as a powerful tool for studying development of the brain's network architecture. In recent years, this methodology has been applied to the human brain in utero, opening a window into a period of functional development that was otherwise inaccessible. Studying fetal fMRI has the potential to illuminate the nature and manner in which the brain's network architecture is initially assembled, affording powerful new insights into neurodevelopmental origins [1, 2, 3, 4]. Despite this potential, progress has been slow due, in part, to the lack of image analysis tools tailored for fetal imaging data. Though many tools and software packages exist for fMRI analysis, these tools were designed with adult and child data in mind and encounter specific problems when applied to fetal functional data. In particular, these tools are not set up to handle encasement of the head inside a heterogeneous tissue compartment, high degree of variation in image properties across subjects, and the extensive position adjustments made by the fetus during a typical scanning session.

Progress made towards improving fetal MRI methodology can broadly be divided into two fields: image acquisition and image post-processing. Image acquisition improvements have occurred mainly with respect to fetal structural MRI, particularly in anatomical (primarily T2 HASTE; Half-Fourier Acquisition Single-shot Turbo spin Echo imaging) and diffusion tensor imaging (DTI) [5, 6]. Advances have been made in inter-slice motion correction and volume reconstruction [7, 8], mapping structural connectivity [9, 10, 11, 12, 13], comparing different MRI signals [14]. These strategies have made it possible to use sparse acquisition sequences, which alleviate movement concerns [15, 16], and enable more sophisticated analytic approaches, such as morphometric [7, 17, 18, 19], cortical folding [20], and cytoarchitectural examinations [21]. In contrast, papers focusing on image post-processing improvements are markedly few and have also primarily focused on structural imaging. Papers suggesting possible solutions for the analysis of fetal functional MRI data are rare [14, 22]. In this work, we focus on methods for improving image post-processing for fetal functional MRI data.

Challenges associated with analysis of fetal fMRI have been discussed in a growing number of studies [23, 24, 25, 4] and reviews [26, 27, 28]. These works have focused on image characteristics: motion, size of the fetal brain, susceptibility artifacts introduced by surrounding maternal tissues, and physiological noise of both mother and fetus. Previous work has highlighted important areas for development, but to our knowledge, no one has proposed a preprocessing pipeline for fetal fMRI and released in the open science framework.

The most time-consuming step in preprocessing fetal fMRI is differentiation of the fetal brain from the surrounding maternal compartment at each acquisition time point. This is achieved by generation of an exemplar mask that marks all in-brain voxels. This mask is critical for the entire preprocessing pipeline and for subsequent activation and/or connectivity analyses. Tools developed to segment the adult brain, such as the Brain Extraction Tool (BET) from FSL [29] and 3dSkullstrip from AFNI [30] are not effective in generating exemplar masks in fetal imaging because the surrounding tissue is more complex and the fetal brain is not in a standard orientation, making it more challenging to identify. As a result, previous studies have relied on manual generation of brain masks [31, 23, 24, 25, 4]. While manual methods are tedious and time consuming, to date, they have been necessary to achieve acceptable standards.

Here, we present an automated approach to the problem of fetal brain segmentation from surrounding tissue. Leveraging a large corpus of manually traced human fetal fMRI masks, we trained a convolutional neural network (CNN) to replace this labor-intensive preprocessing step. CNN's are a powerful tool for effectively identifying complex, non-linear patterns in spatially structured high-dimensional datasets [32]. They are increasingly utilized in image processing applications in both medical and non-medical settings [33, 34]. In the context of fetal brain segmentation, prior work has investigated the application of CNN's to segment fetal structural T2-weighted volumes [35, 36, 37, 38, 16, 39, 40, 41]. These models, however, were developed to segment the fetal brain from anatomical images, and do not translate to functional time series data. Compared to structural data, functional data is significantly lower resolution and, due to movement, requires a larger quantity of individual segmentations. Here, we extend prior work by developing and validating a tool for automatically segmenting the fetal brain from functional MRI data. Ultimately, we connect our auto-masking model with an automated version of a previously manual preprocessing workflow.

All code discussed in this paper, along with a protocol, is available on GitHub (https://github.com/ saigerutherford/fetal-code). This pipeline embodies an initial set of guidelines for fetal fMRI preprocessing, and associated protocols are expected to evolve over time through improvements by the user community in response to new knowledge and innovations in the field. 
bioRxiv preprint doi: https://doi.org/10.1101/525386; this version posted July 13, 2019. The copyright holder for this preprint (which was not

A PREPRINT - JULY 12, 2019

\section{Project Background}

Primary data used for pipeline development were acquired at Wayne State University School of Medicine during the course of projects supported by National Institutes of Health (NIH) awards MH110793 and ES026022. These projects aimed to characterize the development of functional neural systems beginning in utero in relation to prenatal exposures and future neurobehavioral development. Participants were recruited from obstetric clinics located within the Detroit Medical Center. Eligible participants were at least 18 years of age, assessed as having uncomplicated, singleton pregnancies, and had no contraindications for MRI. A physician (with a clinical relationship to eligible patients) initiated contact, and those expressing interest in participating were then introduced to a member of the research team who explained study procedures and answered patient questions. All participants provided written informed consent before undergoing MRI examination. The study protocol was approved by the Human Investigation Committee of Wayne State University.

\section{Methods}

\subsection{Participants and Data}

Resting-state functional MRI was obtained from two cohorts, Wayne State University (WSU) and Yale University. WSU cohort consists of 197 fetuses (gestational age 24-39 weeks, M=30.9, SD=4.2). Twenty-one of these fetuses were scanned at two time points in utero. Both time points are included in this study, however, they are counted as a single subject. WSU fetal MR examinations were performed on a Siemens Verio 3T scanner using an abdominal 4-Channel Flex Coil. Scanning protocols have evolved since the inception of the project in 2012. The majority of data were acquired using echo-planar sequence (TR/TE: 2000/30; 4mm slice thickness, axial, interleaved ascending slice order, 360 volumes) (See Supplementary Materials for all scan parameters). Multi-echo resting-state sequences were also collected in a portion of these subjects (TR/TEs: 2000/18,34,50). The Yale University cohort contains 10 fetuses scanned twice longitudinally (gestational ages 30-36 weeks, M=32.7, SD=1.9). The Yale scanner was a Siemens Skyra 3T using a 32-channel abdominal coil (TR/TE:2000/30; 3mm slices, 32 slices parallel to the bi-commissural plane, 150 volumes). Due to lack of tools for automated segmentation of the fetal brain, research personnel were trained to manually draw fetal brain masks using BrainSuite software [42]. In line with prior work, in the present analysis manually generated brain masks are used to judge the accuracy of automated segmentation methods.

\subsection{Auto-Masking}

\subsubsection{Experimental Pipeline}

Due to multiple manually drawn masks per subject, WSU data were randomly separated at the subject level into training, validation, and test sets with 129,20 , and 48 subjects $(855,102$, and 206 volumes) respectively. The training set was used to optimize the model. The validation set was used to gauge the generalization performance of the network during training and to determine when to stop training. The test set is held out and used only after training was completed to evaluate the performance of the model.

For those interested in using this model on unlabeled data from potentially unseen MRI scanners, we wanted to further demonstrate the transfer ability of our model. We re-trained the CNN a second time by combining both the WSU training and test data (177 subjects/1,066 volumes), and used the same validation set (20 subjects/102 volumes) for determining when to stop training. We tested this CNN model on an additional fetal functional dataset (referred to as the Yale test set) collected in a separate population (New Haven, CT) and on a different MRI scanner. The Yale test set was comprised of 57 volumes from 10 unique subjects.

\subsubsection{Data Preprocessing}

Minimal preprocessing was performed and included removing the image orientation from the image header, resampling and zero padding the images to consistent voxel sizes $(3.5 \times 3.5 \times 3.5 \mathrm{~mm})$ and dimensions $(96 \times 96 \times 37)$. 
A PREPRINT - JULY 12, 2019

\subsubsection{Network Architecture}

The U-Net style CNN network architecture [43] implemented in this pipeline was motivated by prior work in Salehi et al. [41]. The architecture features repeated blocks of $3 \times 3$ convolutions followed by the ReLu activation function assembled into a contracting path, followed by an expanding path. In the contracting path every second convolution is followed by a $2 \times 2$ max pooling operation. In the expanding path, every second convolution is followed by a $2 \times 2$ upsampling operation using nearest-neighbor interpolation. Every other feature map in the contracting path is concatenated along the depth dimension to the corresponding map in the expanding path, which helps the network learn the appropriate location of the output mask. The final layer is convolved with two $1 \mathrm{x} 1$ filters to produce an output mask with channels equal to the number of output classes.

The network separates 3D image volumes into 2D axial slices and operates on each slice independently. We chose to implement a 2D rather than 3D network in order to reduce computational costs. This model includes steps for converting raw NIFTI images into a format readable by the network, and steps for converting the output of the network into a NIFTI-formatted, 3D brain mask.

The model was implemented using Tensorflow (version 1.4.1). Training and testing of the network were performed using a GPU, but CPU testing times are were also evaluated to provide an additional point of reference.

\subsubsection{Training Procedures}

During training, the weights in a CNN are minimized with respect to a loss function that determines how well the network is learning from the training data. We optimized our network with respect to per-pixel cross entropy, with weights determined using the Adam Optimizer [44]. Adam is a first-order gradient method that updates the weights adaptively based on previous and current gradients. Even using an adaptive optimizer, we found that using a learning rate decay improved performance. The initial learning rate was set to 0.0001 with exponential decay rate of 0.9 , applied every 10,000 batches. The model was trained until performance no longer improved on the validation set. In addition, we augmented the $2 \mathrm{D}$ axial slices in the training data through 90-degree rotations and horizontal and vertical flips. While augmentations were done on 2D slices, the same rotations and flips were applied to all slices in order to preserve the 3D shape. These augmentations capture the non-standard orientation of the brain in fetal volumes.

\subsubsection{Evaluation}

The evaluation process was performed over multiple steps. First, we evaluated our network's ability to mask the fetal brain using the dice coefficient. The dice coefficient is the most common evaluation metric for testing segmentation. It measures the percent overlap between two regions: the predicted brain region and the true brain region. It is defined between 0 and 1, where 0 means there is no overlap between the two regions, and 1 means the two regions are identical. We also report the Jaccard index, Hausdoff surface distance [45], sensitivity (true positive rate: brain voxels are correctly identified as brain), and specificity (true negative rate: nonbrain voxels are correctly identified as nonbrain) of our network on the WSU and Yale held-out test sets. Detailed mathematical definitions of these metrics can be found in the supplemental material and guidelines for the choices of these evaluation metrics are described in Taha et al [46].

After training, we calculated dice coefficients for all auto-masks that have a manually drawn complement, though we report values only for volumes in the test data, as performance within the train and validation datasets does not reflect model performance on new data.

\subsection{Comparison to other methods}

In addition, to aid in evaluation of obtained evaluation metrics, we performed a secondary analysis to demonstrate that current methods for adult brain extraction perform poorly when applied to fetal data. We used the Brain Extraction Tool (BET) implemented in FSL, 3dSkullstrip from AFNI, and the fetal anatomical U-Net from Salehi et al. (2017), enabling comparison of approach efficacy. Of note, evaluation metrics can be improved by separating testing data into challenging versus non-challenging images [41], but that approach is not favored and not used here as this diminishes the representativeness of estimates when applied across complex and varied data sets. 
bioRxiv preprint doi: https://doi.org/10.1101/525386; this version posted July 13,2019 . The copyright holder for this preprint (which was not certified by peer review) is the author/funder, who has granted bioRxiv a license to display the preprint in perpetuity. It is made available under aCC-BY-NC 4.0 International license.

A PREPRINT - JULY 12, 2019

Table 1: Summary of all functional data collected at Wayne State University and preprocessed using the proposed automated preprocessing pipeline. The amount of time spent creating brain masks for all available data by automated methods is compared to the amount of time it would take to manually create brain masks.

\begin{tabular}{|l|l|}
\hline Total number of subjects & 248 \\
\hline Number of subject scanned longitudinally & 163 \\
\hline Total number of visits & 411 \\
\hline Total number of runs & 777 \\
\hline Total number of masked volumes & 190,056 \\
\hline Time to auto-mask all volumes & 10.6 hours \\
\hline Time to manually mask all volumes & 22 years \\
\hline
\end{tabular}

\subsection{Failure Analysis}

After model performance was evaluated, we conducted a failure analysis to discover patterns of intrinsic image characteristics that may influence auto-masking performance. First, we examined the relationship between the dice coefficient and gestational age. Next, we examined the effect of varying the number of augmentations in the training dataset. Finally, we evaluated whether image artifacts, brain size ratio (brain volume relative to the entire image volume), or position of the brain in the center of imaging space influence model performance by qualitatively examined images with dice coefficients below 0.9 .

\subsection{Application of the auto-masks}

Auto-masks were generated for all available data, summarized in table1 We note that the number of subjects reported in table 1 is higher than the number of subjects reported in the auto-mask model train/validation/test split. This is due to the time-consuming nature of manual brain masking and thus 51 subjects had available raw data to process but no manually drawn brain masks available. Also many subjects who did have manually drawn masks, had many more un-masked volumes. Visual inspection was used to confirm accuracy and quality of every auto-mask volume within a subject's time-series, and a pass/fail scale was used. Auto-masks are output as spatial probability estimates, wherein voxel values equal to one correspond to highest probability of being brain. Failed auto-masks are flagged and discarded. Probability map brain masks were then clustered, thresholded, and binarized. These steps are taken in order to discard small, non-brain clusters that may have been included in the probability map brain mask. Binarized masks were then resampled back into subject native space where they were applied to the native image using an image multiplier, resulting in segmented brain volumes corresponding to each fetal fMRI data timepoint.

\subsection{Other Preprocessing}

In addition to segmentation of the fetal brain from surrounding maternal tissue, fetal imaging preprocessing requires a number of additional steps: motion denoising, realignment of volumes within a time series, and group-level normalization to a fetal template. All of these steps are challenging because frame-to-frame displacement is elevated in fetal studies, and the fetal brain is typically not in a single standard orientation.

In prior studies by our group, a reference frame from each quiescent period was chosen to be masked. The mask would then be applied to every volume within the low movement period, not only the volume it was drawn on. Due to the time-consuming nature of manual masking, it was not feasible to mask every volume. A central goal of auto-masking is to be able to mask a full time-series in a fraction of the time it takes to manually draw a mask for a single volume. Realigning all volumes within a time series aids in automatically identifying low movement periods that are usable for further activation or connectivity analyses. Volumes in low movement periods are identified by the realignment parameters that are output as a text file and can also be visualized from the saved realignment plots. Typical realignment of fMRI data is done on full time series using the middle volume (in time) as a reference volume. Due to notably high movement across a fetal time series, using a single reference volume for realignment may not 
be an optimal approach. We selected the MCFLIRT FSL realignment tool [29]. While this tool still uses a single reference volume, it estimates a linear transformation between volume $n$ and the reference volume and then uses this transform as the starting point to estimate the $n+1$ to reference transform. These transformation matrices are applied to each volume of the full time series to produce a new data set comprised of realigned volumes. This step also produces a text file and plot that summarize the six rigid-body realignment parameters across time, which can be subsequently used in identification of motion outliers and motion censoring in later processing stages. Here, we applied the fsl_motion_outliers routine as a data-driven means of defining periods of high and low fetal movement.

After masking and realignment, time-series data are converted into a 4-dimensional file, moved into group template space (for multi-subject averaging) using linear warping, and spatially smoothed. Flexibility is built into the pipeline such that the user is able to define whether data are normalized to a common reference template, or alternatively, to age-specific fetal templates, see Serag et al [18]. A linear normalization is implemented via FLIRT [29]. After normalization, all volumes are spatially smoothed with a user-specified Gaussian kernel. Importantly, all software tools used within this preprocessing pipeline (TensorFlow, Python, AFNI, FSL) are free and open-source. All commands can be implemented in a shell script, which can be run from the command line.

\subsection{Quality Checking}

While this methodology employs fully automated techniques for preprocessing fetal resting-state fMRI data, manual quality assurance processes are necessary at key transition points throughout the pipeline. Specifically, our standard process includes initial review of raw time-series data, screened as a movie. Initial inclusion criteria are that the brain is in the field of view and unobstructed by artifacts, and that within the time series, there are periods of minimal fetal movement. We exclude data not meeting these criteria. However, the majority of Wayne State University data passes this stage because long scan durations are used, and fetuses rapidly cycle through quiescent states.

Additional steps in the quality control protocol are implemented after auto-masking, realignment, and normalization. At these stages timeseries data are again visually inspected to assure that no errors were introduced during these stages of preprocessing. Several parameters that are automatically generated during the pipeline should be used in complement to manual quality checking. These parameters include realignment parameters, motion plots, and metrics from the fsl_motion_outliers command. After the time-series has been realigned, calculating the dice coefficient between consecutive volumes could also provide a metric of data quality - a dice coefficient equal to one represents two perfectly overlapping (or realigned) images.

\section{Results}

\subsection{Auto-masking performance}

Our CNN auto-mask model learned to accurately discriminate fetal brain from surrounding structures in fetal brain fMRI images. We evaluated the model on two held-out test sets. Applied to the Wayne State University (WSU - 206 volumes from 48 unique subjects) and Yale (57 volumes from 10 unique subjects) test cohorts, the models achieved a per-volume average dice coefficient of 0.94 and 0.89 , respectively. The CNN's performance in terms of dice coefficient, jaccard coefficient, Hausdorff surface distance, sensitivity, and specificity across both test sets is summarized in Table 2 . Figures 1 and 2 provide examples of agreement between manual and auto-masks in both test sets.

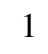

\subsection{Comparison to adult auto-masking tools}

As a point of reference for the dice coefficient achieved by our method, we performed an additional analysis using several existing auto-masking tools: Brain Extraction Tool (BET), 3dSkullstrip, and the fetal anatomical U-Net. Applied to the same test set data, as expected, these tools performed significantly worse. Evaluation metrics are reported 
bioRxiv preprint doi: https://doi.org/101101/525386; this version posted July 13,2019 . The copyright holder for this preprint (which was not certified by peer review) is the author/funder, who has granted bioRxiv a license to display the preprint in perpetuity. It is made available under aCC-BY-NC 4.0 International license.

A PREPRINT - JULY 12, 2019

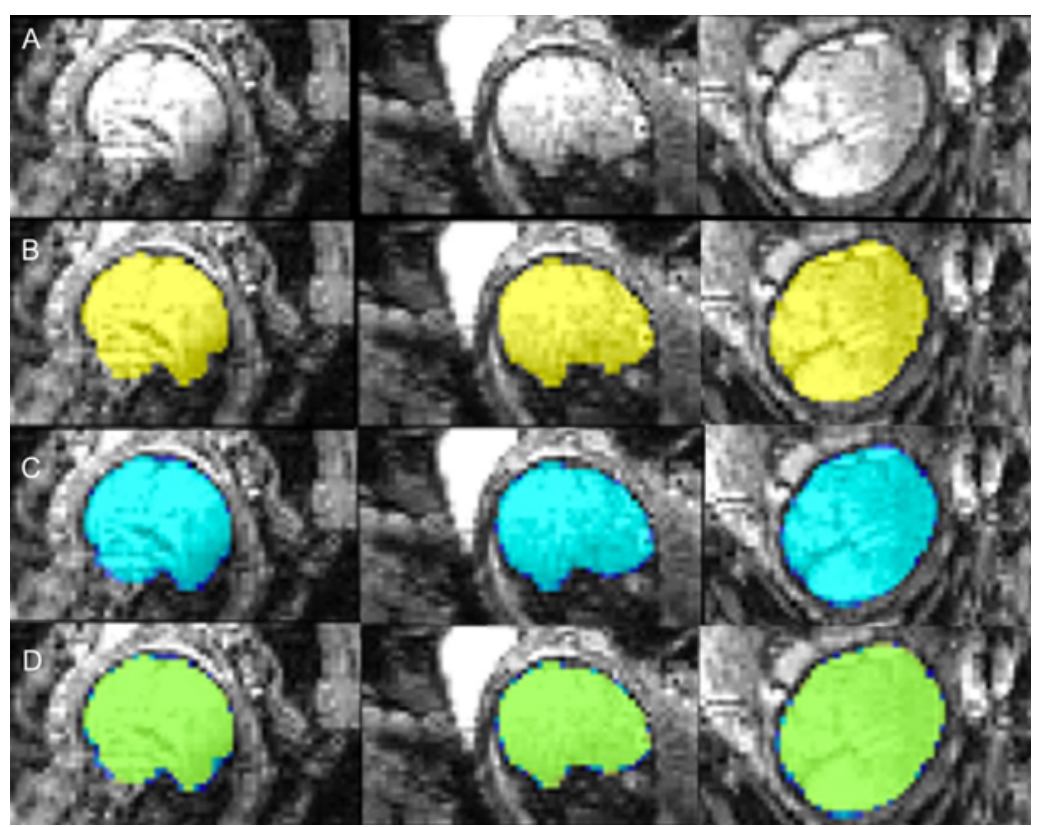

Figure 1: Comparison of manual and automated masks in WSU cohort. A) Raw volume; B) Hand drawn mask; C) Auto mask; D) Conjunction of hand drawn (yellow) and auto (blue) masks, overlap between hand and auto masks shown in green. Data collected in Detroit, MI at Wayne State University.

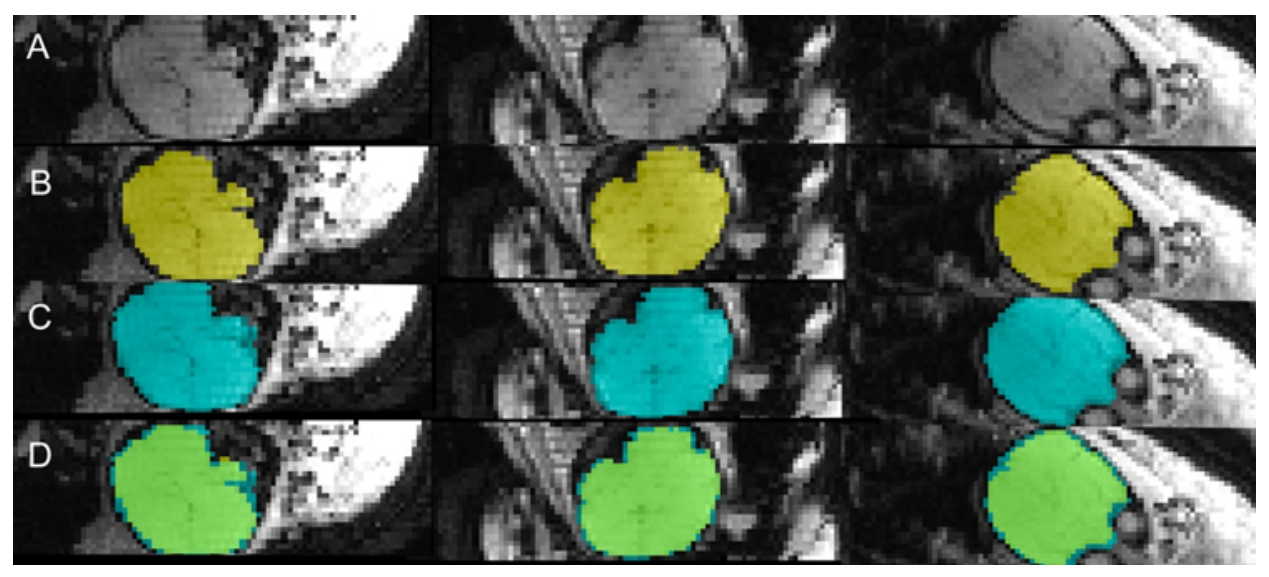

Figure 2: Comparison of manual and automated masks in Yale cohort. These results demonstrate the auto-mask model's ability to transfer to data collected in different populations and/or scanners than the original data used to train the model. A) Raw volume; B) Hand drawn mask; C) Auto mask; D) Conjunction of hand drawn (yellow) and auto (blue) masks, overlap between hand and auto masks shown in green. Data collected in New Haven, CT at Yale University. 
bioRxiv preprint doi: https://doi.org/10.1101/525386; this version posted July 13,2019 . The copyright holder for this preprint (which was not certified by peer review) is the author/funder, who has granted bioRxiv a license to display the preprint in perpetuity. It is made available under aCC-BY-NC 4.0 International license.

A PREPRINT - JULY 12, 2019

Table 2: Performance of auto-mask model and existing masking software evaluated in two independent test sets from Wayne State University (WSU) and Yale University. These values represent the mean and (sd).

\section{WSU Yale}

\begin{tabular}{|l|l|l|l|l|l|c|}
\hline & Auto-mask & \multicolumn{1}{|c|}{ BET } & \multicolumn{1}{|c|}{ 3dSS } & Auto-mask & \multicolumn{1}{c|}{ BET } & \multicolumn{1}{c|}{ 3dSS } \\
\hline Dice & $0.94(0.067)$ & $0.22(0.13)$ & $0.24(0.10)$ & $0.89(0.13)$ & $0.22(0.06)$ & $0.25(0.08)$ \\
Jaccard & $0.89(0.069)$ & $0.13(0.086)$ & $0.14(0.07)$ & $0.82(0.13)$ & $0.13(0.03)$ & $0.15(0.05)$ \\
HD(mm) & $12.11(22.4)$ & $112.6(36.7)$ & $103.3(26.0)$ & $19.25(14.5)$ & $95.2(30.1)$ & $92.8(24.2)$ \\
Sensitivity & $0.90(0.04)$ & $0.13(0.08)$ & $0.14(0.07)$ & $0.84(0.12)$ & $0.12(0.03)$ & $0.15(0.05)$ \\
Specificity & $0.99(0.0007)$ & $0.99(0.003)$ & $0.99(0.002)$ & $0.99(0.002)$ & $0.99(0.004)$ & $0.99(0.002)$ \\
\hline
\end{tabular}

in Table 2 and examples of the masks generated using these tools are shown in figure 3 . The fetal anatomical U-Net masks were empty in most cases, and therefore the evaluation metrics failed and are not reported in Table 2 These results highlight that areas of the maternal compartment have high contrast boundaries and varied image intensity creating serious challenges for standard masking routines and severely compromise performance.

3

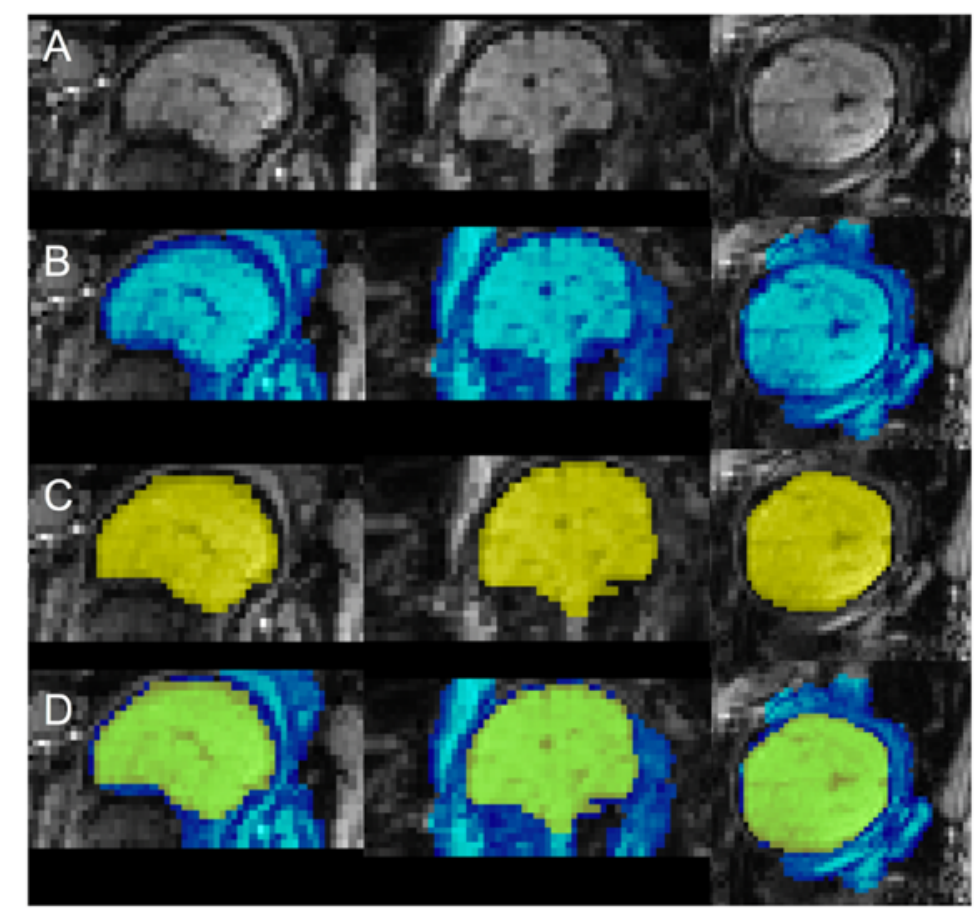

Figure 3: Limitations of the existing toolkit for automated brain masking. A) Raw volume; B) BET mask; C) Hand drawn mask; D) Conjunction of hand drawn (yellow) and BET (blue) masks. The BET masks do not properly capture the fetal brain's boundary.

\subsection{Age and Data Quality Failure Analysis}

Examination of the effect of fetal age on performance of the auto-mask algorithm revealed a significant positive association between dice coefficient and gestational age $(r=0.14, p=0.02)$, jaccard index and gestational age 
bioRxiv preprint doi: https://doi.org/10.1101/525386; this version posted July 13, 2019. The copyright holder for this preprint (which was not certified by peer review) is the author/funder, who has granted bioRxiv a license to display the preprint in perpetuity. It is made available under aCC-BY-NC 4.0 International license.

A PREPRINT - JULY 12, 2019

$(r=0.15, p=0.01)$, and sensitivity and gestational age $\left(r=0.24, p=1 \times 10^{-3}\right)$. There were not significant correlations between gestational age and specificity or gestational age and Hausdorff surface distance. This effect is demonstrated in Figure 4 . This relationship may result from older fetuses having larger brain volumes that intrinsically have higher effective image resolution. The found highly statistically significant relationships between mask volume and dice coefficient $\left(r=0.23, p=2 \times 10^{-3}\right)$, mask volume and jaccard coefficient $\left(r=0.27, p=1.53 \times 10^{-5}\right)$, mask volume and sensitivity $\left(r=0.26, p=1.88 \times 10^{-5}\right)$, and unsurprisingly between mask volume and gestational age $\left(r=0.84, p=1.44 \times 10^{-68}\right)$. We also found that significant aliasing, particularly phase wrap-around negatively impacted auto-mask performance (example shown in Figure 5p), and that the algorithm also performed more poorly for images in which the brain had a large displacement from the image origin. This observation also may explain why the performance improved with an increasing number of augmentations in the training dataset.

4
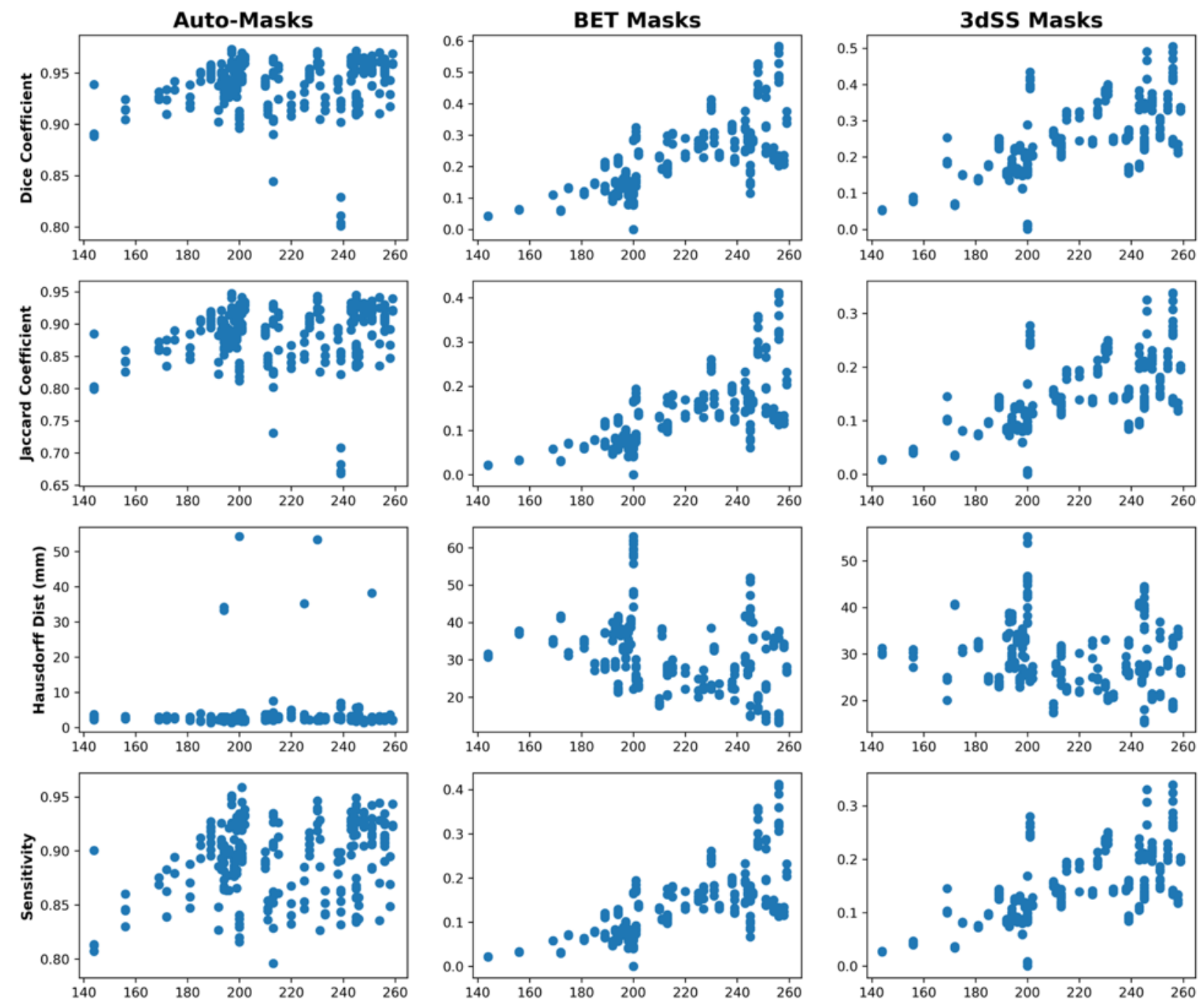

Figure 4: Evaluation of auto-masking model. The relationships between fetal gestational age (in days) at scan is shown on the x-axes and performance of auto-masking in the WSU test set (48 subjects, 206 volumes) y-axes. We calculated the evaluation metrics on a per-volume basis. However, the values shown here are on a per-subject basis in order to examine the relationships with age. There were significant correlations between gestational age and dice coefficient ( $r=0.14, p=0.02)$, jaccard coefficient $(r=0.15, p=0.01)$, and sensitivity $\left(r=0.24, p=1 \times 10^{-3}\right)$.

There were not significant correlations between gestational age and specificity or Hausdorff surface distance. 
bioRxiv preprint doi: https://doi.org/10.1101/525386; this version posted July 13,2019 . The copyright holder for this preprint (which was not certified by peer review) is the author/funder, who has granted bioRxiv a license to display the preprint in perpetuity. It is made available under aCC-BY-NC 4.0 International license.

A PREPRINT - JULY 12, 2019

5

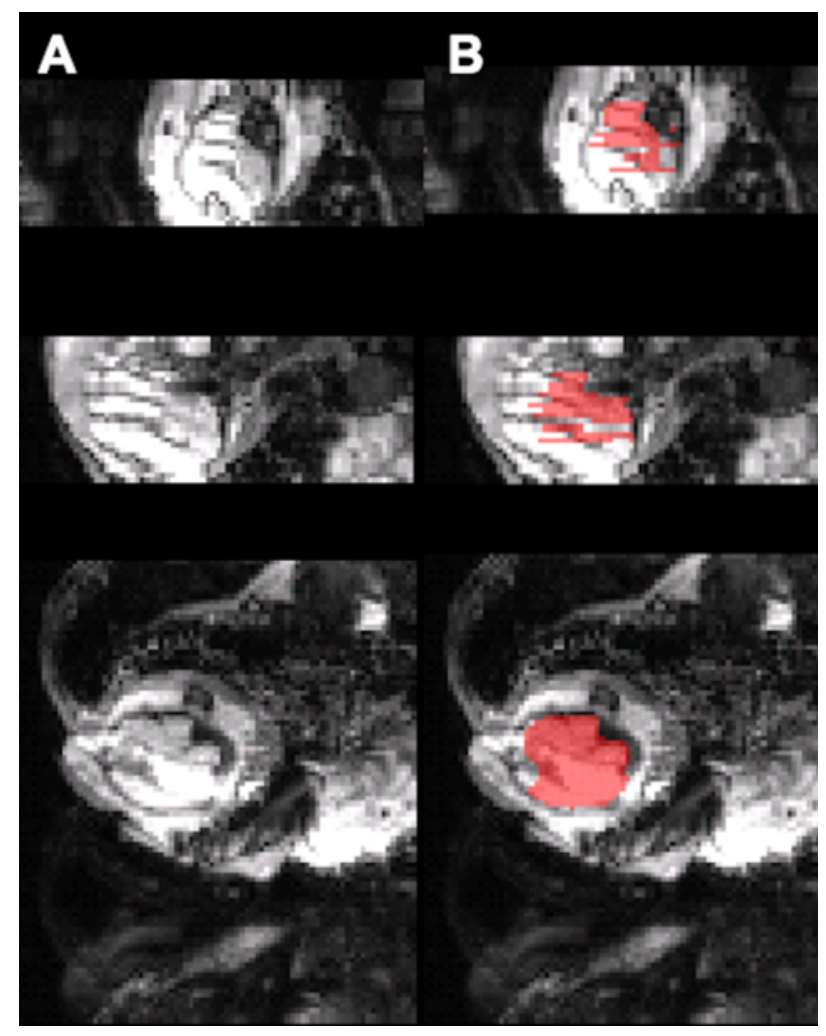

Figure 5: Example of auto-masking failure. A) Raw volume with severe artifacts present. B) Auto-mask that failed to capture a large portion of the fetal brain.

\subsection{Computational Time and Hardware}

An often-noted property of deep learning models is their ability to substantially surpass human speed in completing complex tasks. Our auto-masking model illustrates this acceleration. The training time refers to the wall-clock time it took our CNN model to converge to a set of weights that minimize the dice coefficient on the validation set. Training was stopped after signs of overfitting were observed, that is, performance on the validation set was no longer increasing. The total training time of the model was 3 hours and 46 minutes on a GeForce GTX 1080 Ti GPU. Testing time refers to the time it takes to run the CreateMask.py script in order to load the input raw volume (nifti file type to numpy array conversion) and output a predicted auto-mask. Additionally, we report the time to create auto-masks for all volumes within a subject's time series. As some potential users of these preprocessing methods may not have access to GPU computing resources, we report testing time in GPU as well as CPU environments. These testing times are summarized in Table 3 .

\subsection{Other Preprocessing}

An additional benefit of our approach for auto-masking all individual volumes is that multiple realignment strategies are now possible. After the fetal brain has been extracted, the time series data can enter more typical preprocessing steps for which child/adult tools have been developed. The main difference when applying these tools is that the fetal brain is commonly in a non-standard orientation, and fetal data exhibits substantially increased head motion.

To address the first challenge, we incorporated realignment using MCFLIRT [29] into the pipeline. This method uses the preceding volume to provide an initial realignment estimate for the current volume. The idea behind this 
bioRxiv preprint doi: https://doi.org/10.1101/525386; this version posted July 13, 2019. The copyright holder for this preprint (which was not certified by peer review) is the author/funder, who has granted bioRxiv a license to display the preprint in perpetuity. It is made available under aCC-BY-NC 4.0 International license.

A PREPRINT - JULY 12, 2019

Table 3: Estimated computing times of all preprocessing steps. The time to run the full pipeline is for a single subject with 360 volumes. Testing GPU was a NVIDIA Quadro P6000.

\begin{tabular}{|l|l|l|}
\hline & GPU & CPU \\
\hline Auto-mask a single volume & $0.2 \mathrm{~s}$ & $2.5 \mathrm{~s}$ \\
Auto-mask an entire time series & $1.2 \mathrm{~m}$ & $15 \mathrm{~m}$ \\
Realignment & - & $5 \mathrm{~m}$ \\
Normalization & - & $7 \mathrm{~m}$ \\
Time to run full pipeline & $13.2 \mathrm{~m}$ & $27 \mathrm{~m}$ \\
\hline
\end{tabular}

choice is that there is likely to be high spatial displacement between volumes 10 and 100 because they were collected 180 seconds apart, but less displacement between volumes 10 and 11 collected just 2 seconds apart. With regard to the problem of elevated head motion, errors introduced by movement cannot, at present, be fully corrected in fMRI time series. This fact necessitates application of stringent criteria for retaining only low-motion volumes to ensure data integrity, which is the approach taken by most studies to date [2, 3, 1, 4, 47]. We recommend two criteria for retaining low motion volumes: 1) framewise displacement less than $0.5 \mathrm{~mm}$, and 2) number of consecutive volumes reaching those criteria must be 10 or more. The latter rule reduces the number of breaks in the time series introduced by the data elimination scheme. These criteria await systematic testing against alternative schemes. An overview of the entire suggested preprocessing stream is provided in Figure 6

6

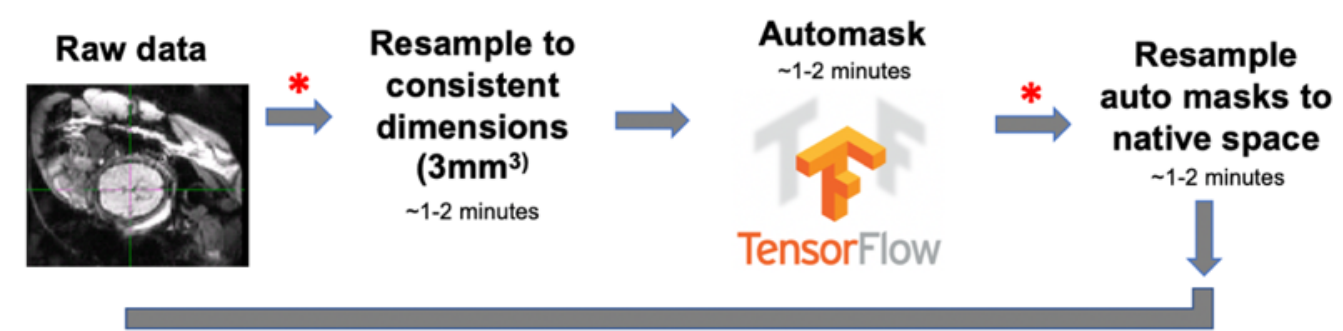<smiles>[I-]</smiles>

Apply masks to raw images $\sim 2-3$ minutes

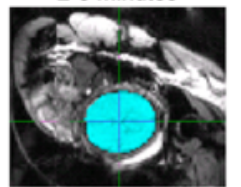

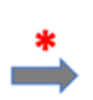

Realign \& identify low motion periods

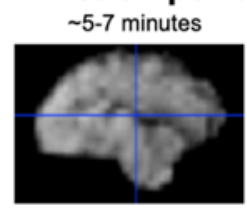

Normalize to age matched template \& smooth

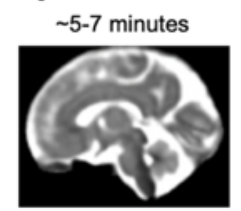

Figure 6: Overview of proposed processing pipeline. All steps in the proposed preprocessing stream are shown, with red asterisk representing where visually quality checking data is recommended. This workflow can be run as shell scripts from the command line.

\section{Discussion}

Fetal functional MRI is an emerging field with great potential to improve understanding of human brain development. The number of papers published in this area has seen a 5-fold increase since 2010. Methodologies for processing these complex data sets, however, have not kept pace, and the absence of a standard publicly available processing pipeline 
for these data has been especially notable. Here we address this gap and present a solution to the most cumbersome processing step, localization and extraction of the fetal brain from surrounding tissue for each volume of a functional time series. This is a necessary step in processing human fetal fMRI data and until now, it has been a rate-limiting factor in accurate, automated processing of fetal fMRI BOLD timeseries data. This model was built by pairing a set of 1,241 hand-drawn fetal 3D brain masks with a deep learning algorithm, a U-Net convolutional neural network. Pipeline code, and documentation are made available through GitHub (https://github.com/saigerutherford/fetal-code). Training, validation, and test data sets (raw volumes, hand drawn masks, and auto-masks) are available through request to Moriah Thomason. It is hoped that release of an easy-to-use, efficient, validated auto-masking pipeline will reduce barriers for new labs to enter this area, while also providing experienced labs opportunities for further optimization of their independently developed approaches.

Recent pioneering work by Salehi and colleagues (2017) established that deep learning approaches can be effective in fetal structural brain segmentation. However, fetal functional imaging presents a distinct set of constraints, and therefore requires a different solution. In particular, the inherently lower resolution and contrast of functional timeseries data, and the 4-dimensional nature of the data ( $3603 \mathrm{D}$ volumes per subject), make this a more challenging problem. Here we applied CNN methods to the largest fetal fMRI data set reported to date, 207 fetuses, and derived a novel method for accurate and reliable segmentation of the fetal brain from surrounding maternal tissues within a fraction of a second, $94 \%$ accuracy in 0.2 seconds. These encouraging results are partially attributable to the large set of manually traced human fetal fMRI masks used to train the CNN, consistent with studies showing the correlation between CNN performance and the size of the training data [48, 49].

The auto-masking model exhibited signs of strong generalizability, which is particularly important given known biases in CNN models trained on imaging datasets from a single site/population [50, 51]. First, the trained CNN correctly classified data at two held out sets with performance, $94 \%$ and $89 \%$, very similar to the training data. This finding suggests that the $\mathrm{CNN}$ is robust to variations in experimental procedures, scanner settings, and populations studied. In addition, the training images were drawn from a wide fetal age-range, which should also enhance generalizability across fetal samples encompassing different ages.

Our aim in this project goes beyond brain segmentation; we sought to construct an automated version of a previously manual full preprocessing pipeline that is standardized but flexible, and readily deployable across multiple data sources. Thus, our pipeline begins with an auto-masking step, then leverages existing algorithms (FSL, AFNI) that assist with applying the auto-masks, frame to frame alignment, normalization to a user-defined template, and smoothing. The user is referred to publicly available multi-age fetal brain templates [18], and is able to easily configure the tool to modify or eliminate steps. The flexibility of our code allows for potential users to mix and match the portions of this pipeline they wish to use. For example, a user could choose an alternative realignment algorithm as the first step then apply the auto-masking step. The open construction of the tool will allow incorporation of future processing advances, such as surface-based registration and additive motion correction strategies.

Of note, large scale, often multi-center, projects are becoming the new norm, and these require validated, standardized processing pipelines of the kind that we have developed. The Developing Human Connectome Project provides just one example of a large-scale study that includes a fetal functional MRI component [52, 53], and many more large-scale fetal fMRI initiatives will likely emerge in the coming years.

Our work has several limitations. First, deep learning methods perform classification in high dimensional space, and consequently, results can be a "black box" with little opportunity for interpretation of axes [54]. However, this limitation should be viewed in the context of our goal: to automatically perform a task that can take trained individuals many hours to perform manually. As such, we are less interested in understanding the mechanisms of computer-based brain masking, and instead focus on algorithm performance in out of sample data sets.

Another limitation is that we use direct warping, or normalization, of functional data to a group-averaged anatomical template. It is not clear that alternatives would improve registration significantly, but one might expect registration to subject-specific anatomy, then to template space, to be a preferred approach. The challenge of this approach is that obtaining high-quality subject-specific high-resolution anatomical images presents a different set of challenges that have been addressed elsewhere [55]. Prior studies of fetal anatomical development [56] demonstrate that even when trained experts apply the most advanced techniques to these data, there is still significant data loss and image blurring where motion effects, image artifacts, or lack of tissue contrast compromise data quality. This example extends to other parts of the pipeline, where alternative optimized preprocessing strategies could be used. However, the objective for this work is not to serve as a final fetal functional MRI preprocessing endpoint, but as a backbone upon which further development can follow. 
bioRxiv preprint doi: https://doi.org/10.1101/525386; this version posted July 13, 2019. The copyright holder for this preprint (which was not certified by peer review) is the author/funder, who has granted bioRxiv a license to display the preprint in perpetuity. It is made available under aCC-BY-NC 4.0 International license.

A PREPRINT - JULY 12, 2019

A final limitation is that ours is not a fully automated pipeline as it requires human supervision and quality checking at several stages, which in turn requires a certain quantity of time and level of expertise from the human supervisor. Fortunately, however, the level of involvement, and associated expertise required, required is limited, and includes looking for overt errors when viewing processed images as a continuous movie, which takes approximately 1-5 minutes per functional run. Assuming the full time for running this pipeline is 15-30 minutes, including human effort, this is a 60 -fold time reduction over prior methods, with manual tracing in particular requiring extensive time and substantial expertise [4, 47].

In sum, in this work, we leverage deep learning methods in the largest sample of fetal fMRI data published to date to address the challenging brain segmentation problem in fetal fMRI. We unite our novel auto-masking tool with other preprocessing steps to initialize the first complete open-source solution to preprocessing raw fetal functional MRI timeseries data.

\section{Acknowledgements}

The authors thank Sahi Karra, Nedda Elewa, Tarek Bazzi, Tahir Khan, Nourhan Hamadi, Bryan Turman, Allison Li, Imran Sheikh, and Sophia Neuenfeldt for their time spent manually generating fetal brain masks, Pavan Jella for assistance in data acquisition, and Lauren Grove for the mentoring and advice while writing this manuscript. The authors also thank participant families who generously shared their time.

\section{Author Contributions}

Conceptualization: SR, MA; Methodology: SR, PS, MA, JH,; Formal Analysis: SR, PS, MA; Data Curation: SR, JH, DS; Writing - Original Draft: SR, PS, JH, MT, CS; Writing - Reviewing and Editing; SR, PS, MA, JH, MV, JW, DS, MT, CS; Visualization: SR, JH; Supervision: MV, JW, MT, CS; Funding Acquisition: MT.

\section{References}

[1] V. Schöpf, G. Kasprian, P. C. Brugger, and D. Prayer. Watching the fetal brain at 'rest'. International Journal of Developmental Neuroscience, 30(1):11 - 17, 2012.

[2] András Jakab, Ernst Schwartz, Gregor Kasprian, Gerlinde M. Gruber, Daniela Prayer, Veronika Schöpf, and Georg Langs. Fetal functional imaging portrays heterogeneous development of emerging human brain networks. Frontiers in Human Neuroscience, 8:852, 2014.

[3] András Jakab, Gregor Kasprian, Ernst Schwartz, Gerlinde Maria Gruber, Christian Mitter, Daniela Prayer, Veronika Schöpf, and Georg Langs. Disrupted developmental organization of the structural connectome in fetuses with corpus callosum agenesis. NeuroImage, 111:277-288, May 2015.

[4] Marion I. van den Heuvel, Elise Turk, Janessa H. Manning, Jasmine Hect, Edgar Hernandez-Andrade, Sonia S. Hassan, Roberto Romero, Martijn P. van den Heuvel, and Moriah E. Thomason. Hubs in the human fetal brain network. Developmental Cognitive Neuroscience, 30:108-115, April 2018.

[5] Oualid M. Benkarim, Gerard Sanroma, Veronika A. Zimmer, Emma Muñoz-Moreno, Nadine Hahner, Elisenda Eixarch, Oscar Camara, Miguel Angel González Ballester, and Gemma Piella. Toward the automatic quantification of in utero brain development in 3d structural MRI: A review. Human Brain Mapping, 38(5):2772-2787.

[6] Anat Biegon and Chen Hoffmann. Quantitative magnetic resonance imaging of the fetal brain in utero: Methods and applications. World Journal of Radiology, 6(8):523-529, August 2014.

[7] Ali Gholipour, Caitlin K. Rollins, Clemente Velasco-Annis, Abdelhakim Ouaalam, Alireza Akhondi-Asl, Onur Afacan, Cynthia M. Ortinau, Sean Clancy, Catherine Limperopoulos, Edward Yang, Judy A. Estroff, and Simon K. Warfield. A normative spatiotemporal MRI atlas of the fetal brain for automatic segmentation and analysis of early brain growth. Scientific Reports, 7(1):476, March 2017.

[8] Colin Studholme. Mapping Fetal Brain Development In Utero Using Magnetic Resonance Imaging: The Big Bang of Brain Mapping. Annual Review of Biomedical Engineering, 13(1):345-368, July 2011. 
[9] Emi Takahashi, Rebecca D. Folkerth, Albert M. Galaburda, and Patricia E. Grant. Emerging cerebral connectivity in the human fetal brain: an MR tractography study. Cerebral Cortex (New York, N.Y.: 1991), 22(2):455-464, February 2012.

[10] Austin Ouyang, Tina Jeon, Susan M. Sunkin, Mihovil Pletikos, Goran Sedmak, Nenad Sestan, Ed S. Lein, and Hao Huang. Spatial Mapping of Structural and Connectional Imaging Data for the Developing Human Brain with Diffusion Tensor Imaging. Methods (San Diego, Calif.), 0:27-37, February 2015.

[11] Anqi Qiu, Susumu Mori, and Michael I. Miller. Diffusion Tensor Imaging for Understanding Brain Development in Early Life. Annual Review of Psychology, 66(1):853-876, January 2015.

[12] Limei Song, Virendra Mishra, Minhui Ouyang, Qinmu Peng, Michelle Slinger, Shuwei Liu, and Hao Huang. Human Fetal Brain Connectome: Structural Network Development from Middle Fetal Stage to Birth. Frontiers in Neuroscience, 11, 2017.

[13] Minhui Ouyang, Jessica Dubois, Qinlin Yu, Pratik Mukherjee, and Hao Huang. Delineation of early brain development from fetuses to infants with diffusion MRI and beyond. NeuroImage, April 2018.

[14] Sharmishtaa Seshamani, Anna I. Blazejewska, Christopher Gatenby, Susan Mckown, Jason Caucutt, Manjiri Dighe, and Colin Studholme. Comparing consistency of R2* and T2*-weighted BOLD analysis of resting state fetal fMRI. In Medical Imaging 2015: Biomedical Applications in Molecular, Structural, and Functional Imaging, volume 9417, page 94170N. International Society for Optics and Photonics, March 2015.

[15] Sharmishtaa Seshamani, Xi Cheng, Mads Fogtmann, Moriah E. Thomason, and Colin Studholme. A Method for handling intensity inhomogenieties in fMRI sequences of moving anatomy of the early developing brain. Medical Image Analysis, 18(2):285-300, February 2014.

[16] Ahmed Serag, Gillian Macnaught, Fiona C. Denison, Rebecca M. Reynolds, Scott I. Semple, and James P. Boardman. Histograms of Oriented 3d Gradients for Fully Automated Fetal Brain Localization and Robust Motion Correction in 3T Magnetic Resonance Images. BioMed Research International, 2017, 2017.

[17] Maria Kuklisova-Murgasova, Paul Aljabar, Latha Srinivasan, Serena J. Counsell, Valentina Doria, Ahmed Serag, Ioannis S. Gousias, James P. Boardman, Mary A. Rutherford, A. David Edwards, Joseph V. Hajnal, and Daniel Rueckert. A dynamic 4d probabilistic atlas of the developing brain. NeuroImage, 54(4):2750-2763, February 2011.

[18] Ahmed Serag, Paul Aljabar, Gareth Ball, Serena J. Counsell, James P. Boardman, Mary A. Rutherford, A. David Edwards, Joseph V. Hajnal, and Daniel Rueckert. Construction of a consistent high-definition spatio-temporal atlas of the developing brain using adaptive kernel regression. NeuroImage, 59(3):2255-2265, February 2012.

[19] Feng Shi, Pew-Thian Yap, Yong Fan, John H. Gilmore, Weili Lin, and Dinggang Shen. Construction of multiregion-multi-reference atlases for neonatal brain MRI segmentation. NeuroImage, 51(2):684-693, June 2010.

[20] R. Wright, V. Kyriakopoulou, C. Ledig, M. A. Rutherford, J. V. Hajnal, D. Rueckert, and P. Aljabar. Automatic quantification of normal cortical folding patterns from fetal brain MRI. NeuroImage, 91:21-32, May 2014.

[21] Jeremy A. Miller, Song-Lin Ding, Susan M. Sunkin, Kimberly A. Smith, Lydia Ng, Aaron Szafer, Amanda Ebbert, Zackery L. Riley, Joshua J. Royall, Kaylynn Aiona, James M. Arnold, Crissa Bennet, Darren Bertagnolli, Krissy Brouner, Stephanie Butler, Shiella Caldejon, Anita Carey, Christine Cuhaciyan, Rachel A. Dalley, Nick Dee, Tim A. Dolbeare, Benjamin A. C. Facer, David Feng, Tim P. Fliss, Garrett Gee, Jeff Goldy, Lindsey Gourley, Benjamin W. Gregor, Guangyu Gu, Robert E. Howard, Jayson M. Jochim, Chihchau L. Kuan, Christopher Lau, Chang-Kyu Lee, Felix Lee, Tracy A. Lemon, Phil Lesnar, Bergen McMurray, Naveed Mastan, Nerick Mosqueda, Theresa Naluai-Cecchini, Nhan-Kiet Ngo, Julie Nyhus, Aaron Oldre, Eric Olson, Jody Parente, Patrick D. Parker, Sheana E. Parry, Allison Stevens, Mihovil Pletikos, Melissa Reding, Kate Roll, David Sandman, Melaine Sarreal, Sheila Shapouri, Nadiya V. Shapovalova, Elaine H. Shen, Nathan Sjoquist, Clifford R. Slaughterbeck, Michael Smith, Andy J. Sodt, Derric Williams, Lilla Zöllei, Bruce Fischl, Mark B. Gerstein, Daniel H. Geschwind, Ian A. Glass, Michael J. Hawrylycz, Robert F. Hevner, Hao Huang, Allan R. Jones, James A. Knowles, Pat Levitt, John W. Phillips, Nenad Šestan, Paul Wohnoutka, Chinh Dang, Amy Bernard, John G. Hohmann, and Ed S. Lein. Transcriptional landscape of the prenatal human brain. Nature, 508(7495):199-206, April 2014.

[22] D. Scheinost, J. A. Onofrey, S. H. Kwon, S. N. Cross, G. Sze, L. R. Ment, and X. Papademetris. A fetal fMRI specific motion correction algorithm using 2nd order edge features. In 2018 IEEE 15th International Symposium on Biomedical Imaging (ISBI 2018), pages 1288-1292, April 2018.

[23] Moriah E. Thomason, Jesse A. Brown, Maya T. Dassanayake, Rupal Shastri, Hilary A. Marusak, Edgar Hernandez-Andrade, Lami Yeo, Swati Mody, Susan Berman, Sonia S. Hassan, and Roberto Romero. Intrinsic Functional Brain Architecture Derived from Graph Theoretical Analysis in the Human Fetus. PLOS ONE, 9(5):e94423, May 2014. 
bioRxiv preprint doi: https://doi.org/10.1101/525386; this version posted July 13, 2019. The copyright holder for this preprint (which was not certified by peer review) is the author/funder, who has granted bioRxiv a license to display the preprint in perpetuity. It is made available under aCC-BY-NC 4.0 International license.

A PREPRINT - JULY 12, 2019

[24] Moriah E. Thomason, Lauren E. Grove, Tim A. Lozon, Angela M. Vila, Yongquan Ye, Matthew J. Nye, Janessa H. Manning, Athina Pappas, Edgar Hernandez-Andrade, Lami Yeo, Swati Mody, Susan Berman, Sonia S. Hassan, and Roberto Romero. Age-related increases in long-range connectivity in fetal functional neural connectivity networks in utero. Developmental Cognitive Neuroscience, 11:96-104, February 2015.

[25] Moriah E. Thomason. Structured Spontaneity: Building Circuits in the Human Prenatal Brain. Trends in Neurosciences, 41(1):1-3, January 2018.

[26] Amy L. Anderson and Moriah E. Thomason. Functional plasticity before the cradle: A review of neural functional imaging in the human fetus. Neuroscience \& Biobehavioral Reviews, 37(9, Part B):2220-2232, November 2013.

[27] Marion I. van den Heuvel and Moriah E. Thomason. Functional Connectivity of the Human Brain in Utero. Trends in Cognitive Sciences, 20(12):931-939, December 2016.

[28] Ashley J. Robinson and M. Ashraf Ederies. Fetal neuroimaging: an update on technical advances and clinical findings. Pediatric Radiology, 48(4):471-485, April 2018.

[29] Mark Jenkinson, Peter Bannister, Michael Brady, and Stephen Smith. Improved optimization for the robust and accurate linear registration and motion correction of brain images. Neurolmage, 17(2):825-841, October 2002.

[30] Robert W. Cox. AFNI: software for analysis and visualization of functional magnetic resonance neuroimages. Computers and Biomedical research, 29(3):162-173, 1996.

[31] Moriah E. Thomason, Maya T. Dassanayake, Stephen Shen, Yashwanth Katkuri, Mitchell Alexis, Amy L. Anderson, Lami Yeo, Swati Mody, Edgar Hernandez-Andrade, Sonia S. Hassan, Colin Studholme, Jeong-Won Jeong, and Roberto Romero. Cross-Hemispheric Functional Connectivity in the Human Fetal Brain. Science Translational Medicine, 5(173):173ra24-173ra24, February 2013.

[32] Y. Lecun, L. Bottou, Y. Bengio, and P. Haffner. Gradient-based learning applied to document recognition. Proceedings of the IEEE, 86(11):2278-2324, November 1998.

[33] Michael Egmont-Petersen, Dick de Ridder, and Heinz Handels. Image processing with neural networks-a review. Pattern recognition, 35(10):2279-2301, 2002.

[34] Matthew D. Zeiler and Rob Fergus. Visualizing and Understanding Convolutional Networks. In Computer Vision - ECCV 2014, Lecture Notes in Computer Science, pages 818-833. Springer, Cham, September 2014.

[35] Tobias Klinder, Hannes Wendland, Irina Wachter-Stehle, David Roundhill, and Cristian Lorenz. Adaptation of an articulated fetal skeleton model to three-dimensional fetal image data. In Medical Imaging 2015: Image Processing, volume 9413, page 94130Q. International Society for Optics and Photonics, March 2015.

[36] Mark Ison, R. Donner, E. Dittrich, Gregor Kasprian, Daniela Prayer, and Georg Langs. Fully automated brain extraction and orientation in raw fetal MRI. In Workshop on Paediatric and Perinatal Imaging, MICCAI, pages 17-24, 2012.

[37] Antonios Makropoulos, Serena J. Counsell, and Daniel Rueckert. A review on automatic fetal and neonatal brain MRI segmentation. NeuroImage, June 2017.

[38] Daphna Link, Michael B. Braginsky, Leo Joskowicz, Liat Ben Sira, Shaul Harel, Ariel Many, Ricardo Tarrasch, Gustavo Malinger, Moran Artzi, Cassandra Kapoor, Elka Miller, and Dafna Ben Bashat. Automatic Measurement of Fetal Brain Development from Magnetic Resonance Imaging: New Reference Data. Fetal Diagnosis and Therapy, September 2017.

[39] Martin Rajchl, Matthew C. H. Lee, Ozan Oktay, Konstantinos Kamnitsas, Jonathan Passerat-Palmbach, Wenjia Bai, Mellisa Damodaram, Mary A. Rutherford, Joseph V. Hajnal, Bernhard Kainz, and Daniel Rueckert. DeepCut: Object Segmentation from Bounding Box Annotations using Convolutional Neural Networks. arXiv:1605.07866 [cs], May 2016. arXiv: 1605.07866.

[40] Sébastien Tourbier, Clemente Velasco-Annis, Vahid Taimouri, Patric Hagmann, Reto Meuli, Simon K. Warfield, Meritxell Bach Cuadra, and Ali Gholipour. Automated template-based brain localization and extraction for fetal brain MRI reconstruction. NeuroImage, 155:460-472, July 2017.

[41] S. S. M. Salehi, D. Erdogmus, and A. Gholipour. Auto-context Convolutional Neural Network (Auto-Net) for Brain Extraction in Magnetic Resonance Imaging. IEEE Transactions on Medical Imaging, PP(99):1-1, 2017.

[42] David W Shattuck and Richard M Leahy. BrainSuite: An automated cortical surface identification tool. Medical Image Analysis, page 14, 2002.

[43] Olaf Ronneberger, Philipp Fischer, and Thomas Brox. U-net: Convolutional networks for biomedical image segmentation. CoRR, abs/1505.04597, 2015. 
bioRxiv preprint doi: https://doi.org/10.1101/525386; this version posted July 13, 2019. The copyright holder for this preprint (which was not certified by peer review) is the author/funder, who has granted bioRxiv a license to display the preprint in perpetuity. It is made available under aCC-BY-NC 4.0 International license.

A PREPRINT - JULY 12, 2019

[44] Diederik P. Kingma and Jimmy Ba. Adam: A Method for Stochastic Optimization. arXiv:1412.6980 [cs], December 2014. arXiv: 1412.6980.

[45] Davood Karimi and Septimiu E. Salcudean. Reducing the Hausdorff Distance in Medical Image Segmentation with Convolutional Neural Networks. arXiv:1904.10030 [cs, eess, stat], April 2019. arXiv: 1904.10030.

[46] Abdel Aziz Taha and Allan Hanbury. Metrics for evaluating 3d medical image segmentation: analysis, selection, and tool. BMC Medical Imaging, 15(1):29, August 2015.

[47] Moriah E. Thomason, Dustin Scheinost, Janessa H. Manning, Lauren E. Grove, Jasmine Hect, Narcis Marshall, Edgar Hernandez-Andrade, Susan Berman, Athina Pappas, Lami Yeo, Sonia S. Hassan, R. Todd Constable, Laura R. Ment, and Roberto Romero. Weak functional connectivity in the human fetal brain prior to preterm birth. Scientific Reports, 7:39286, January 2017.

[48] Junghwan Cho, Kyewook Lee, Ellie Shin, Garry Choy, and Synho Do. How much data is needed to train a medical image deep learning system to achieve necessary high accuracy? arXiv:1511.06348 [cs], November 2015. arXiv: 1511.06348.

[49] Abraham Verghese, Nigam H. Shah, and Robert A. Harrington. What This Computer Needs Is a Physician: Humanism and Artificial Intelligence. JAMA, 319(1):19-20, January 2018.

[50] Tatiana Tommasi, Novi Patricia, Barbara Caputo, and Tinne Tuytelaars. A Deeper Look at Dataset Bias. arXiv:1505.01257 [cs], May 2015. arXiv: 1505.01257.

[51] John R. Zech, Marcus A. Badgeley, Manway Liu, Anthony B. Costa, Joseph J. Titano, and Eric K. Oermann. Confounding variables can degrade generalization performance of radiological deep learning models. arXiv:1807.00431 [cs, stat], July 2018. arXiv: 1807.00431.

[52] Kara E. Garcia, Emma C. Robinson, Dimitrios Alexopoulos, Donna L. Dierker, Matthew F. Glasser, Timothy S. Coalson, Cynthia M. Ortinau, Daniel Rueckert, Larry A. Taber, David C. Van Essen, Cynthia E. Rogers, Christopher D. Smyser, and Philip V. Bayly. Dynamic patterns of cortical expansion during folding of the preterm human brain. Proceedings of the National Academy of Sciences, 115(12):3156-3161, March 2018.

[53] Antonios Makropoulos, Emma C. Robinson, Andreas Schuh, Robert Wright, Sean Fitzgibbon, Jelena Bozek, Serena J. Counsell, Johannes Steinweg, Katy Vecchiato, Jonathan Passerat-Palmbach, Gregor Lenz, Filippo Mortari, Tencho Tenev, Eugene P. Duff, Matteo Bastiani, Lucilio Cordero-Grande, Emer Hughes, Nora Tusor, JacquesDonald Tournier, Jana Hutter, Anthony N. Price, Rui Pedro A. G. Teixeira, Maria Murgasova, Suresh Victor, Christopher Kelly, Mary A. Rutherford, Stephen M. Smith, A. David Edwards, Joseph V. Hajnal, Mark Jenkinson, and Daniel Rueckert. The developing human connectome project: A minimal processing pipeline for neonatal cortical surface reconstruction. NeuroImage.

[54] Federico Cabitza, Raffaele Rasoini, and Gian Franco Gensini. Unintended Consequences of Machine Learning in Medicine. JAMA, 318(6):517-518, August 2017.

[55] Colin Studholme. Mapping the developing human brain in utero using quantitative MR imaging techniques. Seminars in Perinatology, 39(2):105-112, March 2015.

[56] Rita G. Nunes, Giulio Ferrazzi, Anthony N. Price, Jana Hutter, Andreia S. Gaspar, Mary A. Rutherford, and Joseph V. Hajnal. Inner-volume echo volumar imaging (IVEVI) for robust fetal brain imaging. Magnetic Resonance in Medicine, 80(1):279-285, 2018. 